'Black In Immuno, Houston, TX, USA.

${ }^{2}$ Departments of Microbiology \& Immunology and Otolaryngology-Head and Neck Surgery, University of California San Francisco, San Francisco, CA, USA.

${ }^{3}$ Immunology Graduate Program, Stanford University School of Medicine, Stanford, CA, USA.

${ }^{4}$ University of Texas MD Anderson Cancer Center UT Health Graduate School of Biomedical Science, Houston, $T X, U S A$.

${ }^{5}$ McGovern Medical School Department of Neurology. Houston, TX, USA.

${ }^{6}$ Precision Immunology Institute, Icahn School of Medicine at Mount Sinai, New York, NY, USA.

${ }^{7}$ Department of Medicine, University of Chicago,

Chicago, IL, USA

${ }^{8}$ Committee on Immunology, University of Chicago,

Chicago, IL, USA

凶e-mail: joel.babdor@ ucsf.edu

https://doi.org/10.1038, s41577-021-00645-5
Academic research institutions, biotechnological companies, funding agencies, research supportive organizations and scientific societies that operate in the field of immunology together constitute our scientific ecosystem. Within this interdependent professional environment, systemic mechanisms and individual behaviours that reflect a collectively exclusionary culture make our professional fields less diverse, and consequently less creative and effective at tackling scientific and medical challenges ${ }^{1-5}$. Thus, there is an imperative for us all to develop new policies and practices to make our field of research a more diverse and welcoming environment. This is essential if we want to achieve the full potential of our research ecosystems: to creatively tackle scientific questions, accelerate discovery and set the stage for important breakthroughs.

The disconnection and disruption introduced by the COVID-19 pandemic has presented us with a unique opportunity to remodel practices and policies, to extend our networks and to make our professional environments more equitable and inclusive. In the past year, multiple grassroots organizations have used tools already at our disposal - namely, social media, videoconferencing software and other digital platforms to empower and connect Black professionals with one another and with the greater community. Although these individual connections do not supersede the need for addressing issues at the systemic level, the interpersonal relationships and community building have been invaluable. As many of these organizations, including Black In Immuno, recently celebrated their first anniversary, there remains ample opportunities and great need for individuals in the research community, in the greater scientific ecosystem and beyond to connect with these networks and engage with the initiatives spearheaded by these organizations to celebrate and amplify the contemporary and historical contributions of Black professionals in our society.

\section{Making new connections}

Black in Immuno is a non-profit organization established in 2020 in the midst of a national reckoning of systemic racism in the US and the COVID-19 global pandemic that disproportionately affected Black individuals due to widespread social disparities. Following the digital practices developed by other grassroots organizations in the Black in X movement, Black in Immuno has used social media and digital platforms to build an unprecedented network of Black immunologists across the globe, showcase its richness, and connect Black Immunologists to the greater scientific ecosystem in the field of immunology. Mirroring our organization's mission, our flagship event - Black In Immuno Week - features scientific talks, panel discussions and social media campaigns to highlight the scientific achievements and journeys of Black immunologists in front of an international audience, to connect Black immunologists with scientists and research communities interested in their work, to inspire the next generations of trainees to pursue careers in immunology, and to give an opportunity to allies in our scientific ecosystem to join in the celebration and amplification of Black immunologists. The inaugural Black In Immuno Week took place 22-28 November, 2020, and attracted more than 300 live attendees from around the globe and more than 1,000 on-demand viewers. Summaries of this celebratory week have been published elsewhere ${ }^{6,7}$, and recordings of the scientific presentations and panels can be found on our YouTube channel.

Social media and digital platforms have a central role in the ability of Black In Immuno Week to offer opportunities for individuals and institutions that are part of our scientific ecosystem to engage in new networking practices. For example, each Black In Immuno Week starts with a 'roll call' on Instagram and Twitter. In 2020, close to 100 Black immunologists introduced themselves using the hashtag \#BlackInImmunoRollCall and shared their research interests, the scientific questions they are 
working on and the methods they use to address them. The 'roll call' is a unique opportunity for individuals and institutions in our scientific ecosystem to connect with Black immunologists, acknowledge their contributions to science, and amplify their work. In addition to social media, Black In Immuno also used multiple digital platforms, such as Zoom, YoutubeLive and Twitch, to host events. In particular, the multiple scientific mini-symposia live-streamed during Black In Immuno Week and retained on our Youtube channel offer another important opportunity for long-lasting amplification of and networking with Black immunologists, even after Black in Immuno Week has passed. These free and open access scientific talk sessions intentionally feature Black graduate students, postdoctoral researchers and early career scientists and give them a platform to share their work with the scientific ecosystem and beyond. Both types of programming present an invitation for individuals to connect - to reach out to collaborate, form mentorship and sponsorship relationships, and keep tabs on a researcher who might be a great candidate for a hiring opportunity.

Indeed, 'connection' is one of the cornerstones of Black in Immuno, and using digital tools to build these connections has been highly effective for our organization. First, a core mission of the organization from its inception has been to enable individuals who identify both as Black and as an immunologist to connect with each other. In less than one year of active campaigning, our organization has connected with hundreds of Black researchers in the field of immunology. Our community mostly consists of academic scientists so far, but we plan to expand our reach to hundreds of additional individuals in the next year by inviting clinicians, industry scientists and undergraduate students. Second, to connect our network of Black immunologists to the wider scientific community in the field of immunology, we propose to break free from the traditional micro-networking practices at conferences and in the field at large that are predominant in science, technology, engineering, mathematics and medicine; these practices are by nature inefficient at connecting minoritized Black researchers and reinforce the marginalization that they experience in their research microenvironment and within the wider scientific ecosystem ${ }^{8,9}$. As an international collective with volunteers from Africa, the Americas and Europe, Black In Immuno's online-only campaigns with both real time components (for example, livestreamed talks) and ongoing components (for example, 'roll call' and social media campaigns) have at their core the ideals of accessibility and global representation, and the nature of digital platforms have allowed individuals to seek out Black In Immuno and to find one another without our organization's direct facilitation.

\section{Expanding connections and engagement}

The second annual Black In Immuno Week (14-20 November, 2021) builds upon these strategies to grow our community of Black professionals and allies in immunology and immunology-related fields. Recognizing both the scientific and medical contributions made by Black individuals and the mistreatment of Black patient populations and their mistrust of medical research, this year's programming on 'From Bench to Bedside' will reach out more deliberately to clinicians and patient advocates while continuing to grow our community of research scientists. In addition to the 'roll call' of Black immunologists and scientific mini-symposia featuring basic, translational and clinical research, expert panels will provide a view on guidelines, practices and policies that can contribute to making our research environments and patient care more inclusive and equitable.

Since last November, we have continued to use social media to engage our community in conversations about their lives, achievements and struggles as Black immunologists, and as a platform to connect Black immunologists with opportunities and resources. We are currently working on a longer-lasting digital platform to house the vibrant but somewhat ephemeral connections that are made on social media, as well as on several opportunities and resources that will support the career development of our community members. We look forward to engagements with and updates to the immunology community as these efforts come to fruition.

Our organization celebrated its first anniversary by obtaining the official status of a non-profit organization. We hope to gain the support of the entire scientific ecosystem to continue and develop our operations. As members of this greater biomedical ecosystem - as patient advocates, physicians and scientists - we have been trained to use the tools at our disposal or create new tools to solve the problems we see in our midst, ameliorate suffering and improve our lives and the lives of others. We invite you to join us at Black In Immuno Week, to connect with Black immunologists, and ultimately to engage in this long overdue process to identify and build new tools to create an inclusive and innovative biomedical community.

1. Swartz, T. H., Palermo, A.-G. S., Masur, S. K. \& Aberg, J. A. The science and value of diversity: closing the gaps in our understanding of inclusion and diversity. J. Infect. Dis. 220, S33-S41 (2019).

2. Eaton, A. A., Saunders, J. F., Jacobson, R. K. \& West, K. How gender and race stereotypes impact the advancement of scholars in STEM: Professors' biased evaluations of physics and biology post-doctoral candidates. Sex Roles 82, 127-141 (2020).

3. Felder, P. On doctoral student development: exploring faculty mentoring in the shaping of African American doctoral student success. Qual. Rep. 15, 455-474 (2010).

4. Freeman, R. B. \& Huang, W. Collaborating with people like me: ethnic coauthorship within the United States. J. Labor Econ. 33, S289-S318 (2015).

5. Valantine, H. A. \& Collins, F. S. National Institutes of Health addresses the science of diversity. Proc. Natl Acad. Sci. USA 112 12240-12242 (2015)

6. Kouame, E. et al. Black in Immuno week: who we are, what we did, and why it matters. J. Immunol. 207, 1941-1947 (2021).

7. Mbiribindi, B. \& Mobley, A. S. Black in Immuno - amplifying, celebrating, and supporting Black voices in immunology. Eur. J. Immunol. 51, 1306-1308 (2021).

8. Foxx, A. J. et al. Evaluating the prevalence and quality of conference codes of conduct. Proc. Natl Acad. Sci. USA 116, 14931-14936 (2019).

9. Newman, M. E. J. The structure of scientific collaboration networks. Proc. Natl Acad. Sci. USA 98, 404-409 (2001).

\section{Acknowledgements}

The authors acknowledge all past and present Black In Immuno organizers and volunteers for their contributions to Black In Immuno programming, organizational development, and amplification. The authors acknowledge the contribution of Heather Caslin to the coordination of opportunities to communicate Black in Immuno's mission.

\section{Competing interests}

The authors declare no competing interests.

\section{RELATED LINKS}

Black In Immuno: https://www.blackinimmuno.org/

Black In Immuno Week: https://www.blackinimmuno.org/registration Black In Immuno YouTube channel: https://www.youtube.com/channel/ UCOPpqVA8dpqjga3TohyjgYg 